\title{
Therapievergleich bei mittelschwerer und schwerer COPD
}

\section{Tiotropium einmal täglich vermag Exazerbationen einer chronisch obs- truktiven Lungenerkrankung besser vorzubeugen als die Gabe von Salmeterol zweimal täglich.}

— In einer randomisierten Studie in 725 Zentren aus 25 Ländern wurden 7376 Patienten mit mittelschwerer bis schwerer COPD rekrutiert. Sie mussten eine Raucheranamnese (mindestens zehn Packungsjahre) und eine $\mathrm{FEV}_{1}$ unter $70 \%$ nach Bronchodilatation haben, ferner mindestens eine Verschlechterung der Erkrankung innerhalb des letzten Jahres, die zur Gabe von Glukokortikoiden oder Antibiotika bzw. zu stationärer Aufnahme geführt hatte. Die Behandlung mit $18 \mu \mathrm{g}$ Tiotropium mittels
Pulverinhalationssystem einmal täglich oder $50 \mu \mathrm{g}$ Salmeterol über ein Dosieraerosol zweimal täglich wurde mittels Double-Dummy-Technik verblindet und erfolgte über ein Jahr.

Tiotropium verlängerte im Vergleich zu Salmeterol das Risiko bis zu einer Exazerbation von 145 auf 187 Tage. Das Risiko für alle Exazerbationen wurde um $17 \%$, für schwere Formen um $28 \%$ (jeweils $\mathrm{p}<0,001)$ reduziert. Auch die Gesamtzahl aller sowie aller schweren Verschlechterungen nahm signifikant um 11 bzw. 27\% ab. Schwere Nebenwirkungen wurden in der Tiotropium- bzw. Salmeterolgruppe bei 545 (14,7\%) und 606 (16,5\%) Patienten, Todesfälle bei 64 $(1,7 \%)$ bzw. 78 (2,1\%) registriert, jeweils ohne signifikanten Unterschied.

\section{Kommentar}

Nachdem die Leitlinien der Fachgesellschaften für die Behandlung der mittelschweren bis schweren COPD die Gabe von langwirkenden Anticholinergika oder Beta-2-Agonisten ohne Präferenz empfehlen, war ein zuverlässiger Vergleich beider Medikamente überfällig. Der Überlegenheit von Tiotropium war unabhängig vom Alter, vom Geschlecht, vom Schweregrad der COPD, vom BMI und von der Anwendung inhalierter Glukokortikoide.

H. HolzGREVE "

- C. Vogelmeier et al.

Tiotropium versus salmeterol for the prevention of exacerbations of COPD. New Engl. J. Med. 364 (2011) 1093-1103 